2. Bartleson, J.D., Cohen, M.D., Harrington, T.M., Goldstein, N.P. \& Ginsburg, W.W. Cauda equina syndrome secondary to long-standing ankylosing spondylitis. Ann Neurol 1983, 14: 662-669.

3. Hassan, I. Cauda equina syndrome in ankylosing spondylitis: a report of six cases. J Neurol Neurosurg Psychiatry 1976, 39: 1172-1178.

4. Gordon, A.L. \& Yudell, A. Cauda equina lesion associated with rheumatoid spondylitis. Ann Intern Med 1973, 78: 555-557.

5. Russell, M.L., Gordon, D.A., Ogryzlo, M.A. \& McPhedran, R.S. The cauda equina syndrome of ankylosing spondylitis. Ann Intern Med 1973, 78: 551-554.

\section{Worsening neurological status in late pregnancy: consider meningioma}

\section{Sir,}

We would like to report a case of worsening neurological status in late pregnancy due to a meningioma. A single 34 year old primigravida was admitted at 35 weeks gestation complaining of depression and right-sided headaches. The depression was of acute onset whereas the headaches occurred 5 weeks ago but had worsened over the previous week. A preliminary diagnosis of an acute depressive disorder was considered by the psychiatrist. She was afebrile and normotensive. The uterine size was equivalent to a 36 weeks gestation with a single live fetus.

As a result of her refusal or inability to eat and the detection of ketonuria, there was rapid infusion of fluids. Subsequently her condition worsened and neurological reassessment detected a left hemiplegia and ptosis with early bilateral papilloedema. A diagnosis of a right cerebral space occupying lesion was made. Intravenous fluids were restricted to 1 litre in 24 hours, and parenteral dexamethasone administered. She improved but with persistence of the left hemiparesis. An elective caesarean section was performed with delivery of a healthy $2,650 \mathrm{~g}$ male infant. There was immediate improvement and by the third post-operative day, she was able to attend to her baby.

One week postpartum, she travelled to nearby Venezuela for magnetic resonance imaging (MRI) scan and angiography of the brain. These revealed a right temporal lobe lesion. Three weeks later, craniotomy removed a right sphenoidal wing oedematous meningioma. Her recovery was uneventful.

The symptoms of headaches, nausea and vomiting are often encountered in pregnancy leading to complacency in the evaluation of neurological systems of pregnant patients. The consideration of an intracranial lesion at this time was extremely remote. In retrospect, it appeared that the liberal infusion of fluids unmasked the cerebral tumour. Her mood improvement after delivery could be explained by the recognized course of meningiomas in pregnancy. They tend to grow rapidly becoming symptomatic close to term. ${ }^{\text {P Progesterone receptors are present }}$ on meningioma tissue ${ }^{2}$ so that under the influence of progesterone they enlarge by fluid retention and enhanced vascularity; similar to that of fibroids. Delivery with fall in progesterone levels led to shrinkage of the neoplasm. Her recovery thereafter makes this hypothesis plausible and is in keeping with findings of others. ${ }^{3}$

Although the diagnosis was not confirmed initially we considered her clinical condition to be of such severity as to require caesarean section, a policy advocated by others. ${ }^{1,2}$ Even though this is the first case to be reported in the West Indies, it is worth noting that the above symptoms occurring especially in the absence of preeclampsia warrants a thorough neurological evaluation. Meningiomas in the Caribbean constitute $21 \%$ of all neurological lesions, they are particularly prevalent in the 31-50 year age group and females outnumber males 1.68 to $1 .{ }^{4}$ Given these statistics, its presence in West Indian women presenting with worsening neurological symptoms in late pregnancy should always be considered.

G.V. Narayansingh S. Ramsewak J.N. Cross R.U. Adam ${ }^{1}$ W. Kissoon ${ }^{1}$ Faculty of Medical Sciences, University of the West Indies and ISan Fernando General Hospital,

Trinidad, West Indies.

\section{References}

1. De Grood, R.M., Beemer, W.H., Fenner, D.E. \& Compton, A.A. A large meningioma presenting as a neurological emergency in pregnancy. Obstet Gynecol 1987, 69: 439-440.

2. Goldberg, M. \& Rappaport, Z.H. Neurosurgical, obstetric and endocrine aspects of meningioma during pregnancy. Israel $J$ Med Sci 1987, 23: 825-828.

3. Roelvink, N.C., Kamphorst, W., Van Alphen, H.A. \& Rao, B.R. Pregnancy - related primary brain and spinal tumours. Arch Neurol 1987, 44: 209-215.

4. Char, C., Cross, J.N. \& Persaud, M.D. Tumours of the central nervous system. Analysis of 476 cases observed at the University Hospital of the West Indies. West Ind Med J 1987, 36: 140-149.

\section{Intra-operative seeding of tumour cells}

\section{Sir,}

A 74 year old heavy smoker presented with chest pain, anorexia, clubbing and an irregular right lung mass on chest X-ray and computed tomographic scan. Bilobectomy was performed and the histology showed adenosquamous carcinoma. Within three months of the operation, two large subcutaneous masses appeared on both the anterior and posterior edges of the thoracotomy scar (Figure 1). Fine needle aspiration revealed malignant

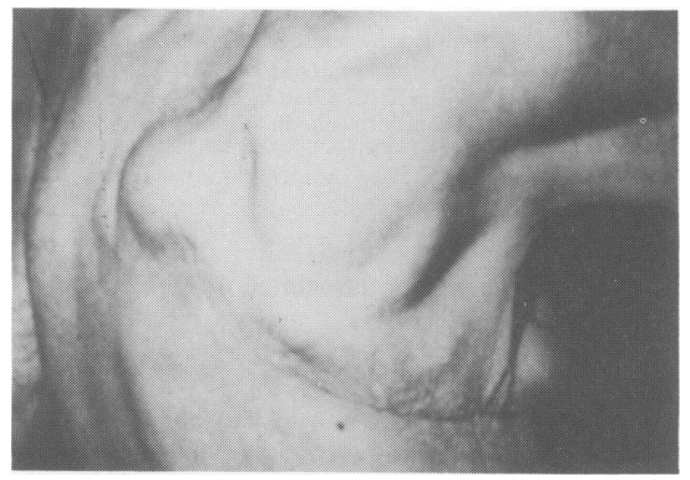

Figure 1 Thoracotomy scar. 
tumour cells. No additional metastases were identified in a thorough search. Such an occurrence may be coincidental but it makes one wonder whether intra-operative seeding of tumour cells should not be a real concern of the surgeon, and what can be done about it?

Ami Schattner, Adi Shani Kaplan Hospital, 76100 Rehovot, Israel.

\section{Carcinoma of the splenic flexure - a case for extended right hemicolectomy?}

Sir,

We have recently managed a patient with a splenic flexure colonic carcinoma, who had an unusual distribution of lymph node metastases. This questions the validity of transverse colectomy for this condition.

The classical treatment of resectable colonic carcinoma has been excision in continuity with its vascular supply in an attempt at reducing local recurrence by removing involved or potentially involved lymph nodes. This is based on Jamieson and Dobson's work ${ }^{1}$ which suggested that the lymphatic drainage of the colon followed its main blood supply. However, blood supply to the splenic flexure has been shown to be somewhat variable. Griffiths $^{2}$ demonstrated that it was supplied by the inferior mesenteric artery via the left colic in $89 \%$ of cases and by the superior mesenteric artery via the middle colic in $11 \%$. He also noted that the middle colic was absent in $22 \%$ of cases. These findings were confirmed by Sierocin$\mathrm{ski}^{3}$ who found in 100 post-mortem dissections that the middle colic vessel supplied the splenic flexure in only 19 . Where the middle colic is absent, the ileocolic supplied the transverse colon and the left colic supplied the splenic flexure.

Goligher $^{4}$ has advocated transverse colectomy for splenic flexure carcinoma, ligating both middle colic at its origin and the ascending branch of the left colic to remove those nodes most likely to be involved.

We report the case of an 83 year old female admitted as an emergency with vomiting and abdominal pain. Plain abdominal X-rays and a gastrografin enema revealed a stenotic lesion at the splenic flexure with complete obstruction. At laparotomy a splenic flexure carcinoma was found and an extended right hemicolectomy performed with an end to end ileocolic anastomosis. The patient made an uneventful recovery and was discharged home.

Histological examination of the specimen showed a moderately differentiated adenocarcinoma of the splenic flexure with one of eight local lymph nodes involved (Duke's stage C Jass Class IV). However, one node at the ileo-colic junction showed adenocarcinomatous deposits.

Aldridge ${ }^{5}$ has shown that patients with a splenic flexure carcinoma have a reduction in 5 year survival rate even when variables such as age, sex, Duke's stage and tumour differentiation were excluded. Three possibilities have been suggested to explain this. Firstly, that the carcinoma may be biologically different, and, secondly, that the host reaction may differ, but these seem unlikely. Lastly, the method of surgical management of the lymph nodes may be inadequate suggesting that an extended right hemicolectomy would be more appropriate. The higher local recurrence rate of tumours of the splenic flexure would seem to support this view.

We suggest, therefore, that an extended right hemicolectomy should be performed for lesions of the splenic flexure. This avoids a colo-anastomosis, and problems due to inadequate bowel preparation and obstruction. It may also remove involved lymph nodes in cases where lymphatic drainage does not follow the classical pattern.

$$
\begin{array}{r}
\text { G.P. Sadler, } \\
\text { R. Gupta, } \\
\text { M.E. Foster } \\
\text { Department of Surgery, } \\
\text { University Hospital of Wales, } \\
\text { Heath Park, } \\
\text { Cardiff, UK. }
\end{array}
$$

\section{References}

1. Jamieson, J.K. \& Dobson, J.F. Lymphatics of the colon with special reference to operational treatment of cancer of the colon. Ann Surg 1909, 50: 1077-1090.

2. Griffiths, J.D. Surgical anatomy of the blood supply of the distal colon. Ann R Col Surg Engl 1956, 51: 241-256.

3. Sierocinski, W. Arteries supplying the left colonic flexure in man. Folia Morphol Waresz 1975, 14: 117-124.

4. Goligher, J.C. Surgery of the Anus, Rectum and Colon, 4th Ed London, Bailliere Tindall, 1980.

5. Aldridge M.L., Phillips, R.K.S., Mittinger, R., Fry, J.S. \& Fielding, L.P. Influence of tumour site on presentation, management and subsequent outcome in large bowel cancer. Br J Surg 1986, 73: 663-670.

\section{Intracranial tuberculoma: paradoxical expansion during medical treatment}

Sir,

Intracranial tuberculomas are only rarely encountered in developed countries. We report a patient whose disease was difficult to diagnose. His clinical situation deteriorated in spite of appropriate administration of antibiotic treatment, and only corticosteroid therapy achieved symptomatic improvement.

A previously healthy 61 year old male began to suffer from nocturnal headaches and rhythmic, clonic contractions of his left lower limb. Blood analysis (including ESR and chest $\mathrm{X}$-ray) was normal. Computerized tomographic (CT) scan showed a hypodense right, parasaggital lesion, with surrounding oedema that enhanced with contrast near the falx, could be seen. A magnetic resonance imaging scan showed a right parasaggital, tumour-like lesion. Corticosteroid treatment was initiated, and symptoms promptly disappeared.

Five cerebral biopsies under stereotactic guide were necessary before noncaseating epithelioid granulomas were found. Appropriate Ziehl-Neelsen staining identified acid-fast bacilli. Daily parenteral treatment with rifampicin, isoniazid, ethambutol, pyrazinamide and dexamethasone was then administered. After six weeks of successful treatment and coinciding with gradual corticosteroid withdrawal, a sudden clinical deterioration including generalized seizures and loss of left lower limb strength was noted. A CT scan showed enlargement of the previous lesion as well as surrounding oedema. Corticosteroid treatment at the initial dose was restored, with the patient improving markedly. 